\title{
AN EVOLUTIONARY ALGORITHM FOR THE DESIGN OF HYBRID FIBER OPTIC-COAXIAL CABLE NETWORKS IN SMALL URBAN AREAS
}

\author{
Pablo Cortés ${ }^{1+}$, Fernando Guerrero ${ }^{1}$, David Canca ${ }^{1}$ and José M García ${ }^{1}$ \\ ${ }^{1}$ Dpto. Ingeniería de Organización, Universidad de Sevilla \\ tpcadesi.us.es
}

\begin{abstract}
Telecommunication is one of the fastest growing business sectors. Future networks will need to integrate a wide variety of services demanding different qualities and capacities from the network. In this paper, network architecture based on hybrid fiber optic-coaxial cable (HFC) is proposed to develop cable integrated telematic services. An evolutionary algorithm is presented to solve the problem in suitable computation times when dealing with real times civil works problems. Finally we present the results over both problem library and real life scenarios.
\end{abstract}

\section{Problem Description}

Telecommunication is one of the fastest growing business sectors of modern Information Technologies. In the past it was enough to get telephone access, but today telecommunications include a vast variety of modern technologies and services. Future networks will need to integrate a wide variety of services demanding different qualities and capacities from the network. In this paper, network architecture based on hybrid fiber optic-coaxial cable (HFC) is proposed to develop cable integrated telematic services.

Nowadays architectures exclusively based on fiber optic, as FTTH (fiber to the home), even its more reduced version FTTC (fiber to the curb), are not profitable because of the amount of investment required. However the fiber optic allows reaching longer distances without regenerating the signal at the same time as transporting great volumes of data at high speed. In this context, fiber optic is specially indicated to constitute backbone links, as well as trunk and distribution links inside the metropolitan network where it substitutes with advantage the coaxial cable. Finally, the coaxial is indicated for the latest network stage correspondent to the subscriber access.

Telecommunication backbones connect with the urban node through the headend, where other analog and digital flows are received and inserted in the metropolitan network. After that a partially meshed fiber optic trunk network connects the head with the primary optical nodes over synchronous digital hierarchy (SDH), attending to the European transmission standard. The distribution network is constructed over fiber optic also, and connects the primary optical nodes with the optical network units (ONU) constituting the latest optic stage. Previously to the ONUs could be located 
secondary nodes acting as splitters with the aim of dividing the downstream signal from one fiber into several fibers. Finally, the feeder network corresponding to the subscriber access stage will be done by means of short length coaxial and lower quality than fiber.

Most of the bibliography based on telecommunication networks attends to large urban areas or inter-municipality communication backbones. Here, we deal with the problem of HFC network deployment in small urban areas. In this context we will have to balance profitability investment with survivability level, and for the smallest urban areas we will have to substitute survivability level to get profitability allowing the network deployment. For this situation small network deployment can be indicated such as star-star architectures, as figure 1 depicts.

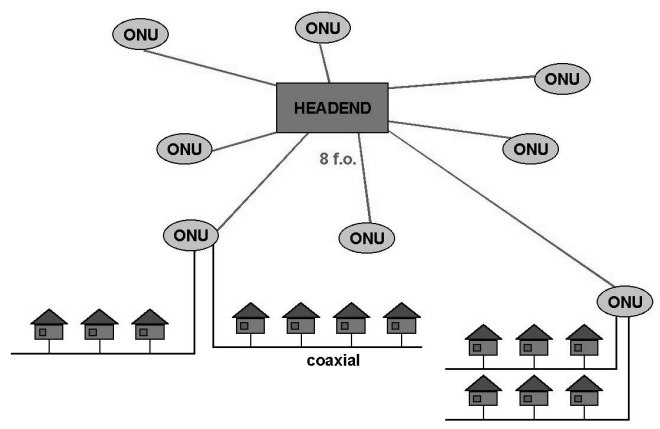

Fig. 1. Simple star architecture

The case can be well suited by the Steiner problem, as we will see in next section.

The rest of the paper deals with the problem formulation in section 2. Section 3 presents an evolutionary algorithm to solve the problem adaptively. Section 4 evaluates the utility of the approach into a problem library and shows the results for a real case corresponding to a municipality in the south of Spain. Finally section 5 summarizes the main results and conclusions.

\section{Problem Formulation}

The problem we have to deal with try to minimize the HFC network deployment cost in a small urban area. We suppose a set of nodes representing the headend and ONUs as known data. The problem of headend and ONUs location has been extensively dealt in the bibliography, examples are [1] and [2]. The potential network where the fibers will be placed is represented by a graph constructed from the urban street network

This situation is depicted by the next figure 2, where headend and ONUs correspond to terminal nodes and the rest of nodes (simple intersections in the urban street network) correspond to Steiner nodes attending to the Steiner problem nomenclature. The arcs represent feasible options where a conduit could be placed. 
The fiber optic placement over the physical network gives place to the logical network displayed in figure 2, in which each of the own fiber optic cables, communicating the headend with the ONU, is laid inside the shared conduits.

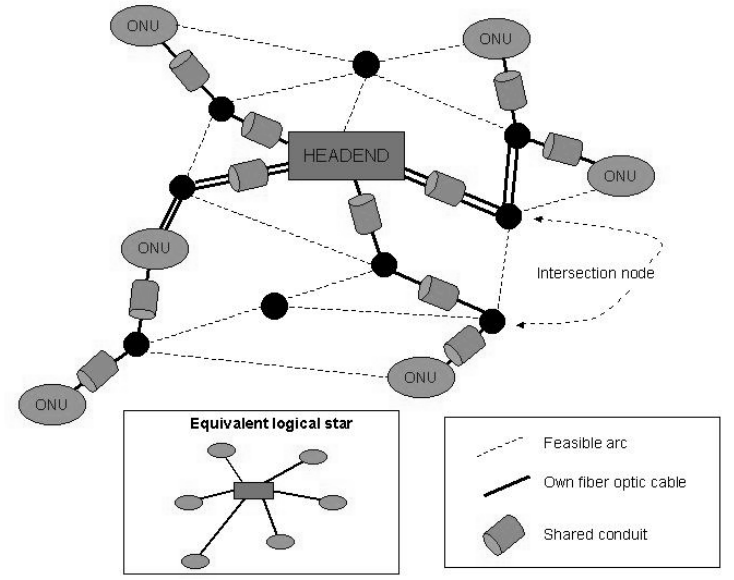

Fig. 2. Logical star network

As the conduit construction is the most relevant part of the telecommunication network setting up, costs can be directly approached by means of the arc lengths. As we have noted, this problem is stated as the Steiner problem in networks that can be summarized as [3]:

- Given: A non-directed graph $G=(N, A)$ with $|\mathrm{N}|$ nodes and $|A|$ arcs with costs $c_{i j}$, for all $(i, j) \in A$, and a subset $I \subseteq N$ with $|I|$ nodes called terminals (in our case headend and ONUs). The rest of the nodes are called Steiner nodes.

- Find: A network $G_{I} \subseteq G$ joining all the terminal nodes in $I$ at minimum cost. This network can include some of the Steiner nodes but has not to include all the Steiner nodes.

The network $G_{I}$ will link the entire ONUs with the headend while the arcs will represent the facilities to place the conduits and lay the cables.

To formulate the problem we make use of the following notation:

\section{Parameters:}

- $\mathrm{N}$ set of nodes in the graph

- A set of arcs in the graph. We will separate the set $A$ into the subsets $A^{+}$for increasing arcs, i.e., if $i<j \Rightarrow(i, j) \subset A^{+}$, and $A^{-}$for decreasing arcs, i.e., if $i>j \Rightarrow(i, j) \subset A$

- I set of terminal nodes in the network, i.e., the headend and ONUs. So $|I|$ represents the number of terminals

- J set of Steiner nodes in the network. So, $|J|$ represents the number of Steiner nodes.

Variables: 
- $\mathrm{y}_{\mathrm{ij}}$ binary variable taking value equal to one if there exits flow through the arc $(i, j)$, zero otherwise.

- $\mathrm{x}_{\mathrm{ij}}$ flow variable, representing the amount of flow through the arc $(i, j)$ in the direction $i \rightarrow j$.

- $\mathrm{x}_{\text {ie }}$ flow variable, representing the amount of flow between the node $i$ and the headend, $e$.

Data:

- $\mathrm{c}_{\mathrm{ij}} \quad$ fixed cost due to the conduit construction between nodes $i$ and $j$.

To model the problem we make use of the multi-injection formulation [4]. This formulation can be analyzed as a weak form of the Steiner problem as different from formulation in [5] that represents an equivalent but stronger form more useful when a branching method have to be implemented.

Model:

$$
\begin{array}{lr}
Z=M I N \sum_{(i, j) \in A^{+}} c_{i j} y_{i j} & \\
s t: & \forall(i, j) \in A^{+} \\
\sum_{j \in I(i)} x_{i j}-\sum_{j \in I(i)} x_{j i}=\left\{\begin{array}{rc}
1 & \forall i \in I \\
0 & \forall i \in J \\
-|I| & i=e
\end{array}\right. \\
|I| \cdot y_{i j} \geq x_{i j}+x_{j i} & \forall(i, j) \in A \\
x_{i j} \geq 0 & \forall(i, j) \in A^{+}
\end{array}
$$

Constraint (1) is the flow balance equation. In the upper part, one unit of flow is sent from each of the ONUs, $i \in I$. In the medium part shows how the inflow equates the outflow at each node Steiner of the network. Finally, the total amount of flow homing into the headend $e$ must be equal to the total amount of flow injected into the network, i.e., the number of ONUs. In the summations, the set $I(k)$ indicates all the incident nodes into the node $i$. Constraint (2) is used to set to one the value of the binary variable $y_{i j}$ when there exists positive flow through the $\operatorname{arc}(i, j)$.

The model uses the $x_{i j}$ variables to obtain a completely connected graph and the $y_{i j}$ variables to evaluate the cost function. The dimension of the final model are $|N|+1 / 2|A|$ constraints, $1 / 2|A|$ integer variables and $|A|$ continuous variables.

\section{Evolutionary Algorithm}

One of the most popular approaches to deal with the Steiner problem is the tree heuristic known to have a worst-case analysis [6]. This approach involves an error not upper than $100 \%$. This is one of the better known bounded heuristic approaches. 
We present here an evolutionary algorithm that makes use of the tree heuristic to present a new algorithm that beats it, guaranteeing the worst case deviation and capable of adapting to the any kind of urban network. This algorithm constructs subnetworks by means of neighborhoods of size/radius $\alpha$ containing, at least, one terminal node. Applying the tree heuristic internally inside the sub-network, a feasible solution is obtained for the local Steiner problem. After that, the method is applied, again, among the different neighborhoods, shaping a Steiner super-tree. The neighborhood size term, characterized in the parameter $\alpha$, turns to critical when an universal application is intended, and when managing with bounded computation times. Here the evolutionary algorithm fits the $\alpha$ parameter allowing the convergence to a good solution.

This new approach makes use of three clear ideas. First, when the number of terminals and the size of the problem are reduced, the local tree heuristic solution will be nearer to the local optimum solution (the heuristic works better with small problems). Second, when the separate sets have to be linked across one only arc, the solution so obtained will express better one of the principal Steiner ideas, a big flow over one arc cost the same that a small flow over the same arc. Finally, as the sum of minimums is always lower or equal than the minimum of the sum, and if the minimums are feasible the sum of minimums is feasible too, then it can be concluded that the worst-case deviation is maintained.

In this context we set as the population individuals any feasible •-value charactering a neighborhood. So the genetic encoding is defined by each •-value implying a different Steiner problem solution. The characteristics for the evolutionary algorithm are as follows.

- Uniform crossover operators. Calculated as the arithmetic average between each pair of $\bullet$-parameters.

- Mutation operator. A mutation process will modify one of the --parameters in the population by setting it to random value between the maximum arc length and the minimum arc length.

- Random parents selection. All the individuals from the size $N$ population are randomly selected. This will enrich the population genetic variety.

- Ranking based replacement. We propose the use of a hypergeometric function to let more probability of replacement to the individuals with worse fitness and less probability of replacement to the individuals with better fitness. So, the individual in ranking position- $i$, have a replacement probability given by $q(1-$ $q)^{i}$, being $q$ the replacement probability of the worst individual.

- Based on population entropy stop criterion. We follow [7] as a good procedure to control the convergence in genetic algorithms. The maximum entropy limitation is given by: $S=\sum_{i=1}^{N} \alpha_{i} \cdot \ln \left(\alpha_{i}\right)$

\section{Results}

All the tests have been run on a $200 \mathrm{MHz}$ PC Pentium MMX workstation. The OR-library available in [8] was used to test the heuristics computationally. Our algorithm was put in competition with the tree heuristic and was compared with 
the available optimum values. Table 1 summarizes the results as well as the error percentage with respect to the optimum solution. The referred parameters in the table (nodes, terminals and arcs) are relative to the results after preprocessing the Winter rules [3], so nodes and arcs are specified with respect to the reduced graph corresponding to each ID problem. Keys tree and eval have been used to refer the tree heuristic and the evolutionary algorithm respectively.

Table 1. Problem library computational results

\begin{tabular}{|c|c|c|c|c|c|c|c|c|c|}
\hline$\overline{I D}$ & Nodes & $\%$ terms & $\operatorname{arcs}$ & Optimum & tree & $\%$ error & eval & $a$ & $\%$ error \\
\hline Stainh1 & 1.2 & 615 & 19 & 82 & 82 & $n n$ & 82 & 2.12 & $n n$ \\
\hline Steinb2 & 15 & 73.3 & 21 & 83 & 85 & 2.4 & 89 & 8 & 7.2 \\
\hline Steinb3 & 20 & 75.0 & 25 & 138 & 138 & 0.0 & 138 & $1-8$ & 0.0 \\
\hline Steinb4 & 40 & 22,5 & 80 & 59 & 62 & 5,1 & 62 & $1-3$ & 5,1 \\
\hline Steinb5 & 39 & 30.8 & 80 & 61 & $\frac{2}{61}$ & 0.0 & 62 & $8-10$ & 1.6 \\
\hline Steinb6 & 45 & 55,6 & 87 & 122 & 126 & 3,3 & 124 & $4-6$ & 1,6 \\
\hline Steinb7 & 22 & 50.0 & 33 & 111 & 112 & 0.9 & 112 & 4 & 0.9 \\
\hline Steinb8 & 26 & 57.7 & 38 & 104 & 105 & 1.0 & 105 & 4 & 1.0 \\
\hline Steinb9 & 27 & 85,2 & 35 & 220 & 221 & 0,5 & 220 & $2-4$ & 0,0 \\
\hline Steinb1 & $\frac{11}{55}$ & 23,6 & 121 & 86 & 91 & 5,8 & 90 & 4 & $\frac{1,6}{4,7}$ \\
\hline Steinb1 & 63 & 30.2 & 129 & 88 & 90 & 2.3 & 90 & $1-10$ & 2.3 \\
\hline Steinb1 & 63 & 57.1 & 125 & 174 & 174 & 0.0 & 174 & $5-10$ & 0.0 \\
\hline Steinb1 & 36 & 38.9 & 56 & 165 & 172 & 4.2 & 172 & 5-14 & 4.2 \\
\hline Steinb1 & 42 & 50,0 & 65 & 235 & 238 & $\begin{array}{l}1,2 \\
1,3\end{array}$ & 238 & 2 & $\begin{array}{l}1,2 \\
1,3\end{array}$ \\
\hline Steinb1 & 47 & 80.9 & 67 & 318 & 321 & 0.9 & 321 & $5-12$ & 0.9 \\
\hline Steinb1 & 77 & 22.1 & 166 & 127 & 137 & 7.9 & 137 & $\frac{1-2: 5-10}{}$ & 7.9 \\
\hline Steinb1 & 74 & $\frac{2.1}{31.1}$ & 153 & 131 & 133 & 1.5 & 133 & 2 & 1.5 \\
\hline Steinb1 & 82 & 54,9 & 165 & 218 & 222 & 1,8 & 222 & $5: 7-10$ & 1,8 \\
\hline Steinc1 & 143 & 3,5 & 260 & 85 & 85 & 0,0 & 85 & $6-9$ & 0,0 \\
\hline Steinc2 & 128 & 7.8 & 234 & 144 & 144 & 0.0 & 150 & 8 & 4.2 \\
\hline Steinc3 & 178 & 42.1 & 295 & 754 & 774 & 2.7 & 775 & 4 & 2.8 \\
\hline Steinc4 & 193 & 52,8 & 314 & 1079 & 1096 & 1,6 & 1086 & 7 & 0,6 \\
\hline Steinc5 & 223 & 80,7 & 341 & 1579 & 1582 & 0,2 & 1581 & 8 & 0,1 \\
\hline Steinc6 & 366 & 1.4 & 837 & 55 & 60 & 9.1 & 55 & 4 & 0.0 \\
\hline Steinc7 & 383 & 2.6 & 866 & 102 & 114 & $\frac{0.1}{11.8}$ & 103 & $7: 10$ & 1.0 \\
\hline Steinc8 & 387 & 20.4 & 867 & 509 & 532 & 4.5 & 529 & 8 & 3.9 \\
\hline Steinc9 & 4 & $\frac{0.4}{29,7}$ & 903 & 707 & $\frac{306}{728}$ & $\frac{4,5}{3,0}$ & $\frac{754}{724}$ & $\frac{0}{7}$ & 2.4 \\
\hline Steinc1 & 427 & 56,7 & 891 & 1093 & 1116 & 2,1 & 1114 & $8-10$ & 1,9 \\
\hline Steinc1 & 499 & 1.0 & 2005 & 32 & 37 & 15.6 & 33 & $7-8$ & 3.1 \\
\hline Steinc1 & 499 & 2.0 & 2065 & 46 & 48 & 4.3 & 46 & $2: 6-8$ & 0.0 \\
\hline Steinc1 & 498 & 16,7 & 2026 & 258 & 273 & 5,8 & 277 & 3 & 7.4 \\
\hline Steinc1 & 499 & 25.1 & 1968 & 323 & 342 & 5.9 & 339 & 8 & 5.0 \\
\hline Steinc1 & 500 & $\frac{0.1}{50.0}$ & 1814 & 556 & 572 & 2.9 & 565 & 3 & 1.6 \\
\hline Steinc1 & 500 & 1.0 & 3517 & 11 & 13 & 18.2 & 12 & $2-4$ & 9.1 \\
\hline Steinc1 & 500 & 2.0 & 3463 & 18 & 20 & 11.1 & 20 & $1-2$ & 11.1 \\
\hline Steinc1 & 500 & 16,6 & 3495 & 113 & 126 & 11,5 & 126 & 1 & 11,5 \\
\hline Steinc1 & 500 & 25,0 & 3349 & 146 & 159 & 8,9 & 159 & $2-4$ & 8,9 \\
\hline Steinc2 & 500 & 50.0 & 3099 & 267 & 268 & 0.4 & 269 & $1: 4$ & 0.7 \\
\hline Steind1 & 272 & 1.8 & 504 & 106 & 107 & 0.9 & 107 & $5-15$ & 0.9 \\
\hline Steind2 & 283 & 3,5 & 519 & 220 & 235 & 6,8 & 228 & $1-3: 8-$ & 3.6 \\
\hline Steind3 & 350 & 42,3 & 585 & 1565 & $\frac{5}{1612}$ & 3,0 & 1600 & $10-14$ & 2,2 \\
\hline Steind4 & 359 & 57,0 & 590 & 1935 & 1970 & $\begin{array}{l}0,0 \\
1.8\end{array}$ & 1969 & $10-14$ & $\begin{array}{l}\frac{L, L}{1.8} \\
\end{array}$ \\
\hline Steind5 & 470 & 80.2 & 708 & 3250 & 3269 & 0.6 & 3268 & 4 & 0.6 \\
\hline Steind6 & 759 & 0,7 & 1730 & 67 & 74 & 10,4 & 71 & $9-10$ & 6,0 \\
\hline Steind7 & 749 & $\begin{array}{l}, 1,3 \\
\end{array}$ & 1722 & 103 & 105 & 1,9 & 103 & 5 & 0,0 \\
\hline Steind8 & 802 & 20.7 & 1778 & 1072 & 1144 & 6.7 & 1140 & $5-6$ & 6.3 \\
\hline Steind9 & 802 & 30.7 & 1769 & 1448 & 1534 & 5.9 & 1528 & 2 & 5.5 \\
\hline Steind1 & 836 & 58.0 & 1781 & 2110 & 2165 & 2.6 & 2163 & 1 & 2.5 \\
\hline Steind1 & 993 & 0,5 & 4442 & 29 & 31 & 6.9 & 29 & 6-10 & 0,0 \\
\hline Steind1 & 1000 & 1,0 & 4437 & 42 & 52 & 23,8 & 43 & $8-10$ & 2,4 \\
\hline Steind1 & 998 & 16.7 & 4354 & 500 & 554 & 10.8 & 549 & 2 & 9.8 \\
\hline Steind1 & $\begin{array}{l}998 \\
9\end{array}$ & 25.1 & 4309 & 667 & 723 & 8.4 & $\begin{array}{l}723 \\
\end{array}$ & 1 & 8.4 \\
\hline Steind1 1 & 996 & 50.0 & 3916 & 1116 & 1150 & 3.0 & 1152 & $4-5$ & 3.7 \\
\hline Steind1 & 1000 & 0.5 & 8048 & 13 & 15 & 15.4 & 13 & $2-4$ & 0.0 \\
\hline Steind1 & 1000 & 1,0 & 8061 & 23 & 30 & 30,4 & 25 & 2 & 8,7 \\
\hline Steind1 & 1000 & 16.7 & 7755 & 223 & 257 & 15.2 & 254 & $2-3$ & 13.9 \\
\hline Steind1 & 1000 & 25.0 & 7552 & 310 & 348 & $\frac{10.5}{12.3}$ & 347 & $\frac{2-4}{2-4}$ & $\frac{11.9}{11.9}$ \\
\hline Steind? & 1000 & $\frac{50.0}{50,0}$ & $\frac{102}{6887}$ & 537 & 546 & $\begin{array}{l}1,0 \\
1.7\end{array}$ & \begin{tabular}{|l|l|}
545 \\
\end{tabular} & $\frac{4}{1-4}$ & 1,5 \\
\hline
\end{tabular}

We have to note at the respect of the results the following considerations in relation with the problem of designing HFC telecommunication networks. Firstly, errors low 
than $5 \%$ have to be considered as successes when we are dealing with conduit installation costs, because of upper deviations in real time civil works will certainly take place. Secondly, for small urban areas graphs upper than 1,000 nodes will not be common, so we can consider the library quite representative in relation with our problem

The average error was estimated in $5.6 \%$ for tree and $3.6 \%$ for eval. Eval reached a total of 10 optimums (42 successes) for 6 optimums (34 successes) in tree case. The maximum error was calculated in $30.4 \%$ for tree and $13.9 \%$ for eval.

We have evaluated our algorithm in real life scenarios also. Here is represented a case study in which the model and algorithm are applied to the Montequinto area. Montequinto is a residential urban area sited near the metropolitan area of Seville, the largest city in the south of Spain, where advanced telecommunication services will probably be welcomed. Montequinto has a population around 25,000 inhabitants.

Figure 3 shows the HFC network solution for the Montequinto area. As primary design decision a simple star architecture was selected to distribute telecommunication services. The figure reveals the conduit physical network, and its equivalent logical star representation.

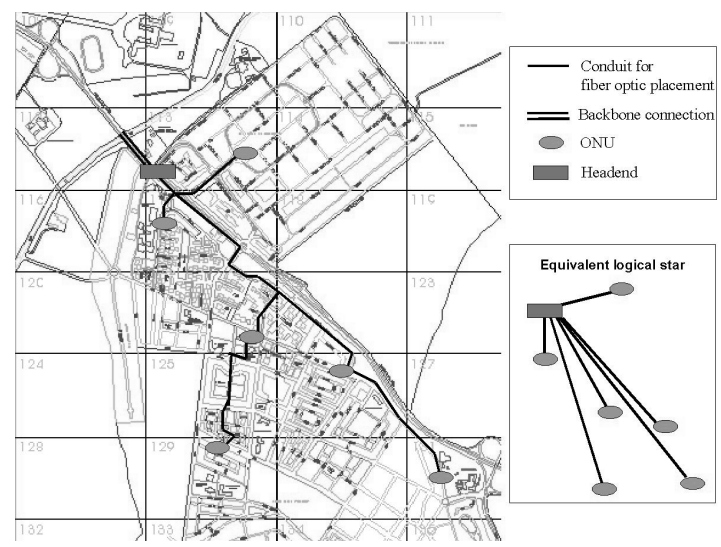

Fig. 3. HFC network in Montequinto urban area

The figure depicts the tree topology corresponding to the conduit network. This tree topology corresponds to a logical simple star when we represent the fiber network. In this small urban area context, a sub-headend, smaller than typical headends and dependent on the Seville headend, has been located near the motorway to provide easy connection between the trunk network and the inter-urban backbone. To distribute the telecommunication services six ONUs are displayed over the municipality. The connection among the sub-headend and the ONUs depicts the optical trunk-distribution network. 


\section{Conclusions}

Most of the bibliography based on distributing telecommunication networks attends to large urban areas or inter-municipality communication backbones. Here, we deal with a small urban area where network deployment has to be not too large trying to maximize the profitability investment. In this context simple star architectures are indicated to broach the situation. Steiner problem is accurate to model the trunk and optical distribution network deployment. A multi-injection formulation is introduced to model the problem as a single commodity flow problem instead of a multicommodity flow problem, reducing the number of constraints and variables. An evolutionary algorithm has been presented showing a good behavior beating traditional generally accepted approaches. The evolutionary algorithm is used to set the neighborhood parameter allowing a universal and adaptive application. Finally, we test the model and algorithm within problem library and illustrate a real life application by means of a case study relative to a residential urban area near to Seville.

\section{References}

1. Belvaux, G., Boissin, N., Sutter, A. and Wolsey L.A.: Optimal placement of add/drop multiplexers: static and dynamic models. Eur J Opl Res 108 (1998) 26-35

2. Sutter, A., Vanderbeck, F. and Wolsey, L.A.: Optimal placement of add/drop multiplexers: heuristic and exact algorithms. Opns Res 46 (1998) 719-728

3. Winter, P.: Steiner problem in networks. Networks 17 (1987) 129-167

4. Cortes, P., Larrañeta, J., Onieva, L. and Garcia, J.M.: Multi-injection model to solve the Steiner problem. In: Sforza, A. (ed.): Simulation and Optimisation in Operations Management. Edizioni Scientifiche Italiane, Napoli (1999) 83-84

5. Wong, R.T.: A dual ascent approach for Steiner tree problems on a directed graph. Math Program 28 (1984) 271-287

6. Magnanti, T.L., Wolsey, L.A.: Optimal trees. In: Ball, M.O. et al (eds.): Network Models. North-Holland, Elsevier (1995) 503-615

7. Grefenstette, J.J.: Incorporating Problem-Specific Knowledge into Genetic Algorithms. In Davis, L. (ed.): Genetic Algorithms and their Applications. Morgan Kaufmann, Los Angeles (1987)

8. Beasley, J.E.: OR-Library. http://mscmga.ms.ic.ac.uk/info.html (2001). 